\title{
Patient-Reported Outcomes in Free-Flap Breast Reconstructive Surgery over Time (PRO-BREST)
}

\author{
Duveken B.Y. Fontein ${ }^{a} \quad$ Melanie Oros ${ }^{a}$ Leonhard Held ${ }^{b}$ Pietro Giovanoli ${ }^{a}$ \\ Andrea L. Pusic ${ }^{c}$ Nicole Lindenblatt ${ }^{a}$ \\ ${ }^{a}$ Department of Plastic Surgery and Hand Surgery, University Hospital Zurich, Zurich, Switzerland; ${ }^{b}$ Epidemiology, \\ Biostatistics and Prevention Institute, University of Zurich, Zurich, Switzerland; 'Division of Plastic Surgery, Brigham \\ and Women's Hospital, Boston, MA, USA
}

\section{Keywords}

Breast reconstruction - Breast cancer - Quality of life ·

Patient-reported outcome measure $\cdot$ Coping

\begin{abstract}
Introduction: Patient-reported outcomes (PROMs) are increasingly relevant to assess surgical quality and guide decisions in breast reconstruction (BR). Satisfaction with outcomes may change as time progresses. We assessed satisfaction in patients who underwent free-flap BR in the last 12 years. Methods: All patients who underwent free-flap BR from 2006 to 2018 were invited to complete the validated BREAST-Q for reconstruction. The BREAST-Q comprises 6 domains covering various aspects of satisfaction. Unadjusted linear regression assessed the relationship between different domains of the BREAST-Q and time since BR. Two-sample $t$ tests assessed differences in satisfaction between patients who underwent $B R \geq 5$ years versus $<5$ years prior. Results: Forty-three women with primary or secondary free-flap BR between 2006 and 2018 were included in the study. Most patients ( $n=33,76.7 \%)$ underwent DIEP flap BR. Overall satisfaction with breasts and with outcomes improved as time since BR increased ( $p=0.031$ and $p=0.017$, respectively). Overall satisfaction with outcomes scored higher in patients with $B R \geq 5$ years prior ( $\geq 5$ years vs. $<5$ years: breast score 88.6 (SD 12.5) versus 66.9 (SD 21.8); $p=0.005)$. Satisfaction with breasts and psychosocial well-being also scored higher in these patients. There was no difference in results between
\end{abstract}

primary and secondary BR. Patients who underwent additional surgery (refinements) reported higher satisfaction with outcomes and abdominal well-being. Conclusions: PROMs concerning satisfaction with breast and with outcomes following BR improve as time since treatment progresses. This study demonstrates that time since diagnosis may be an important factor in satisfaction. It underlines the importance of long-term PROMs related to BR, to help provide patients and health care professionals in decision-making and in managing expectations related to BR.

\section{(c) 2021 The Author(s)}

Published by S. Karger AG, Basel

\section{Introduction}

Breast cancer is the most common invasive cancer among women affecting an increasing number of women worldwide. Breast cancer incidence has been estimated around 89.7 cases per 100,000 women in Western Europe, according to the World Health Organization [1]. Many women diagnosed with breast cancer undergo mastectomy, which can lead to a range of long-term psychosocial problems including distortion of body image, decreased femininity and attractiveness, and reduced sexual desire and pleasure [2]. A study by Beugels and colleagues [3] reported that $50 \%$ of all women who underwent mastec-

Presented at the 55th Congress Swiss Plastic Surgery annual conference (SGPRAEC 2019), St. Moritz, Switzerland.

\section{(C) 2021 The Author(s)}

Published by S. Karger AG, Basel

This is an Open Access article licensed under the Creative Commons Attribution-NonCommercial-4.0 International License (CC BY-NC) (http://www.karger.com/Services/OpenAccessLicense), applicable to the online version of the article only. Usage and distribution for commercial purposes requires written permission.
Correspondence to:

Duveken B.Y. Fontein, duveken.fontein@usz.ch 
tomy experienced negative changes in self-image and sexuality. Breast reconstruction (BR) can facilitate in restoring psychosocial problems associated with mastectomy $[2,4]$. Previously, women reported many reasons for undergoing $\mathrm{BR}$, including the desire to improve body image and restoring feelings of wholeness and integrity [2]. Following BR, women reported higher satisfaction with their reconstructed breast(s) in comparison to women who underwent mastectomy without reconstruction [5-7].

In the last years, breast cancer survival has increased significantly due to early detection and improved treatment and more emphasis is placed on improving psychological outcomes [3, 8-10]. Furthermore, common surgical outcome measures alone such as morbidity, postoperative complications, functional results, and objective measures of cosmetic outcomes do not suffice as outcomes for breast reconstructive surgery $[11,12]$. Since the overall goal of $\mathrm{BR}$ is to improve the appearance of the patient's breast(s) in order to attain a higher quality of life (QoL), patient-reported outcome measures (PROMs) are important markers of successful reconstructive surgery $[13,14]$.

The BREAST-Q for BR is a validated and well-accepted questionnaire for patient-reported satisfaction and QoL assessment [15]. Previous studies investigating patients' long-term QoL and satisfaction have revealed that satisfaction is higher following BR than in mastectomy patients who do not undergo BR $[7,16]$. Complications also play an important role in patient satisfaction following BR $[9,17,18]$. However, studies investigating changes in patient satisfaction over longer periods of time are still relatively lacking, and QoL and satisfaction with outcomes may change as time since reconstructive surgery passes [19]. In this study, we aimed to assess long-term QoL and satisfaction outcomes based on PROMs in postmastectomy patients who underwent free-flap BR between 2006 and 2018.

\section{Methods}

\section{Materials and Methods}

We performed a single-center cross-sectional cohort study investigating the QoL in women after BR. Ethical approval for the study protocol by the Swiss Cantonal Ethics Committee was obtained (BASEC No. 2018-00867). All patients provided written informed consent. The study was designed in accordance with the EQUATOR Network's recommendations on reporting patientreported outcomes in clinical trials [20]. All female patients (18 years or older) who underwent primary or secondary free-flap BR to one or both breasts for any stage breast cancer, benign breast tumors, or for breast cancer prevention at the University Hospital of Zurich between 2006 and 2018 were invited to participate. Patients with other diseases/conditions to the breast (i.e., burns, congenital malformations, or posttraumatic breast deformities), and patients who did not return the questionnaire, did not speak German, or had an invalid address were excluded from this study.

\section{Patients and Data Collection}

Patients were enrolled from the patient and surgical records of the University Hospital of Zurich. Patients who met the inclusion criteria were invited to complete the BREAST-Q questionnaire [12] for BR (postoperative) at home. Non-responders were contacted a second time per telephone. Routinely collected data on patient-, disease-, and treatment-specific characteristics, breastspecific surgical data, complications, and photo documentation was obtained through the hospital's patient records.

\section{Questionnaires}

QoL was measured using the validated BREAST-Q questionnaire for BR module (postoperative) version 2.0, November 2017 (German version) [12]. The questionnaire consists of three QoL domains: 1) psychosocial well-being, 2) sexual well-being, 3) physical well-being, and satisfaction domains: 1) satisfaction with breast, 2) satisfaction with overall outcome, 3) satisfaction with care. Raw data from the BREAST-Q was converted using the Qscore software [12]. For each scale, a summary score (0-100) was constructed using the individual answers from each patient. This score represents the QoL and satisfaction. The higher the score, the greater the satisfaction or the better the QoL.

\section{Statistical Analysis}

Based on standard BREAST-Q scoring procedures, all scales were linearly converted to a $0-100$ scale. For the statistical analysis, the breast scores in relation to time since BR were compared through unadjusted linear regression. In addition, patients were grouped according to time since $\mathrm{BR}(<5$ and $\geq 5$ years prior to receiving the BREAST-Q). We excluded domains pertaining to satisfaction with care due to the risk of recall bias. Two-sample $t$ tests were used to assess differences in QoL between the two groups of patients. The significance level was fixed at $5 \%$. Statistical analyses were performed using IBM SPSS Statistics, version 25 (IBM, Armonk, NY, USA). Frequencies of baseline characteristics between groups were compared using Fisher's exact test in R-Studio (RStudio version 1.3.959, 2009-2020). Unknown or missing data were excluded from the analyses.

\section{Results}

After screening, 114 female patients with postmastectomy, free-flap BR between 2006 and 2018 were invited to participate in this study. Overall response rate was $37.7 \%(n=43)$. Table 1 presents baseline characteristics of patients included in the study. Mean age was 53.8 years (range 33-79 years). Most patients underwent primary $\mathrm{BR}(58.1 \%)$. The mean time since BR was 3.3 years (range $0.3-12.4$ years). The number of responders who underwent $\mathrm{BR}<5$ years ago was $33(76.7 \%)$ versus 10 patients who were treated $\geq 5$ years ago $(23.3 \%)$. The majority of patients underwent DIEP flap BR $(n=33,76.7 \%)$. Other flap types included TRAM, ms-TRAM, SIEA, SGAP, IGAP, and TMG/TUG flaps. Type of reconstruction, free-flap type, and disease site were similar between patients treated $<5$ years and those treated $\geq 5$ years ago. Approximately half of all patients underwent adjuvant hormonal therapy $(55.8 \%)$ and $60.5 \%(n=26)$ underwent adjuvant chemotherapy. Seventeen patients (39.5\%) 
Table 1. Baseline characteristics of patients included in this study

\begin{tabular}{|c|c|c|c|c|}
\hline \multirow[t]{2}{*}{ Baseline characteristics } & \multirow[t]{2}{*}{ All patients } & \multicolumn{2}{|c|}{ Time since breast reconstruction } & \multirow[t]{2}{*}{$p$ value } \\
\hline & & $\geq 5$ years & $<5$ years & \\
\hline Patients & 43 & $12(27.9)$ & $31(72.1)$ & \\
\hline Age (mean, range) & $53.8(33-79)$ & $56.3(49-70)$ & $53.1(33-79)$ & \\
\hline Time since BR (mean, range) & $3.3(0.3-12.4)$ & $7.1(5.0-12.4)$ & $2.1(0.3-4.9)$ & \\
\hline \multicolumn{5}{|l|}{ Site of disease } \\
\hline Left & $23(53.5)$ & $8(66.7)$ & $15(48.4)$ & \multirow[t]{3}{*}{0.39} \\
\hline Right & $17(39.5)$ & $4(33.3)$ & $13(41.9)$ & \\
\hline Bilateral & $3(7.0)$ & $0(0.0)$ & $3(9.7)$ & \\
\hline \multicolumn{5}{|l|}{ Type of reconstruction } \\
\hline Primary & $25(58.1)$ & $7(70.0)$ & $18(54.5)$ & \multirow[t]{2}{*}{0.48} \\
\hline Secondary & $18(41.9)$ & $3(30.0)$ & $15(45.4)$ & \\
\hline \multicolumn{5}{|l|}{ Flap type } \\
\hline DIEP & $33(76.7)$ & $9(75.0)$ & $24(77.4)$ & \multirow[t]{2}{*}{0.87} \\
\hline Other & $10(23.3)$ & $3(25.0)$ & $7(22.6)$ & \\
\hline \multicolumn{5}{|l|}{ NAC reconstruction } \\
\hline Yes & $16(39.0)$ & $6(50.0)$ & $10(34.5)$ & \multirow[t]{2}{*}{0.35} \\
\hline No & $25(61.0)$ & $6(50.0)$ & $19(65.5)$ & \\
\hline \multicolumn{5}{|l|}{ Chemotherapy } \\
\hline Yes & $26(60.5)$ & $5(41.6)$ & $21(67.7)$ & \multirow[t]{3}{*}{0.25} \\
\hline No & $14(32.6)$ & $5(41.6)$ & $9(29.0)$ & \\
\hline Unknown & $3(7.0)$ & $2(16.6)$ & $1(3.2)$ & \\
\hline \multicolumn{5}{|l|}{ Radiotherapy } \\
\hline Yes & $17(39.5)$ & $4(33.3)$ & $13(41.9)$ & \multirow[t]{3}{*}{0.79} \\
\hline No & $22(51.2)$ & $6(50.0)$ & $16(51.6)$ & \\
\hline Unknown & $2(9.3)$ & $2(16.6)$ & $2(6.5)$ & \\
\hline \multicolumn{5}{|l|}{ Hormonal therapy } \\
\hline Yes & $24(55.8)$ & $7(58.3)$ & $17(54.8)$ & \multirow[t]{3}{*}{0.52} \\
\hline No & $15(34.9)$ & $3(0.25)$ & $12(38.7)$ & \\
\hline Unknown & $4(9.3)$ & $2(16.6)$ & $2(6.5)$ & \\
\hline \multicolumn{5}{|l|}{ Additional elective surgery } \\
\hline 0 & $21(50.0)$ & $7(58.3)$ & $14(46.7)$ & \multirow[t]{4}{*}{0.79} \\
\hline 1 & $14(33.3)$ & $3(25.0)$ & $11(36.7)$ & \\
\hline 2 & $6(14.3)$ & $2(16.7)$ & $4(13.3)$ & \\
\hline$\geq 3$ & $1(2.4)$ & $0(0.0)$ & $1(3.3)$ & \\
\hline
\end{tabular}

Data are presented as $n(\%)$ unless indicated otherwise. BR, breast reconstruction; NAC, nipple-areola complex.

Table 2. Unadjusted linear regression outcomes for satisfaction and well-being domains in relation to time since breast reconstruction

\begin{tabular}{lllll}
\hline BREAST-Q domain & Coefficient & $R^{2}$ & $95 \% \mathrm{Cl}$ & $p$ value \\
\hline Satisfaction with breast & 0.33 & 0.11 & $0.22-4.23$ & 0.031 \\
Satisfaction with outcome & 0.37 & 0.14 & $0.59-5.61$ & 0.017 \\
Psychosocial well-being & 0.22 & 0.05 & $-0.64-3.80$ & 0.16 \\
Sexual well-being & 1.08 & 0.02 & $-1.75-3.90$ & 0.45 \\
Physical well-being: chest & 2.03 & 0.08 & $-0.14-4.21$ & 0.066 \\
Physical well-being: abdomen & 3.19 & 0.09 & $-0.22-6.59$ & 0.066 \\
\hline \multicolumn{1}{c}{ Cl, confidence interval. } & & & & \\
\hline
\end{tabular}

had adjuvant radiotherapy. There were no statistically significant differences in baseline characteristics between responding and non-responding patients (data not shown).

Using unadjusted linear regression, scores for overall satisfaction with breasts and satisfaction with outcomes improved in relation to time since BR (regression coefficient: 0.33 and $0.37 ; R^{2} 0.11$ and $0.14 ; p=0.031$ and $p=$ 0.017 , respectively) (Table 2; Fig. 1a, b). There was a trend towards improved physical well-being regarding both the chest and abdomen as time since BR progressed. Results for satisfaction with breasts and satisfaction with out- 
Fig. 1. Scatter plot of satisfaction with breast (a) and outcomes (b) by time since free-flap breast reconstruction.

(a)

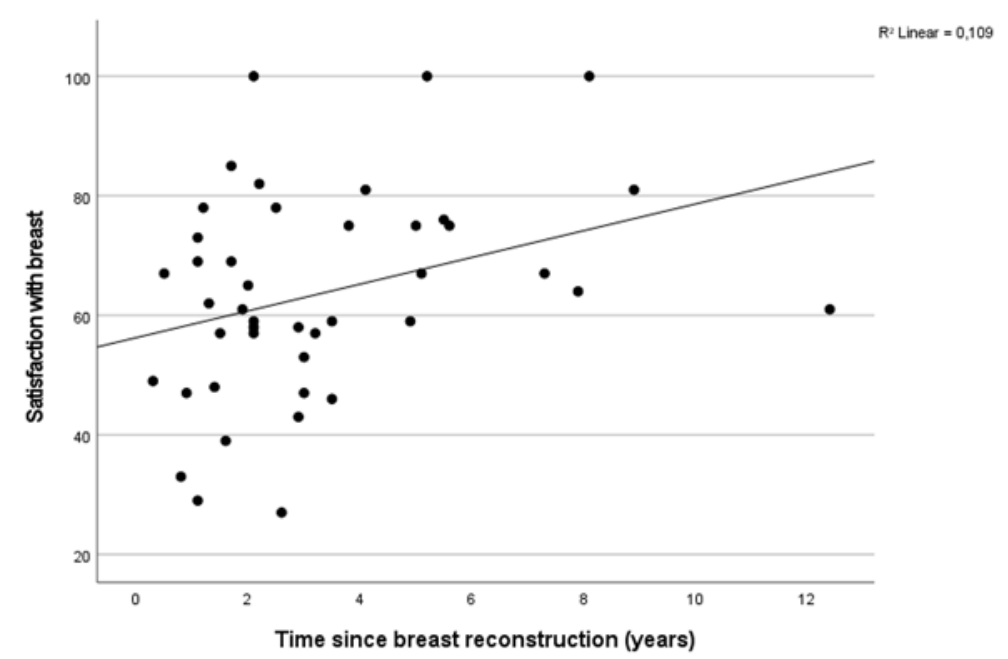

(b)

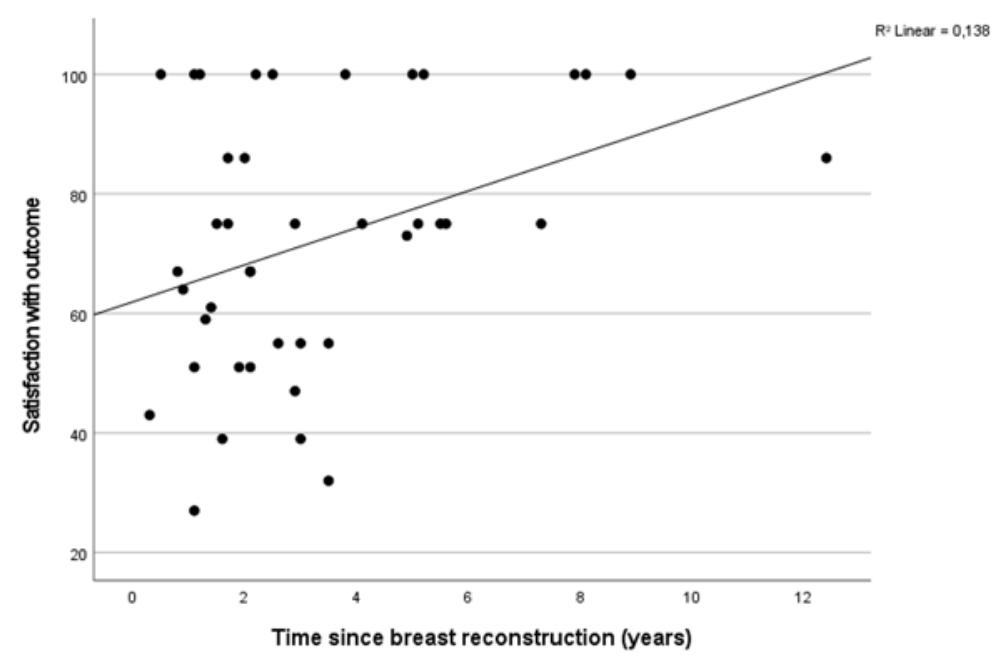

come, as well as psychosocial well-being scored higher in patients who underwent breast reconstructive surgery $\geq 5$ years ago (satisfaction with breasts: $p=0.006$; satisfaction with outcome: $p=0.005$; psychosocial well-being: $p=$ 0.016) (Table 3; Fig. 2). Sexual well-being and physical well-being outcomes did not show differences between the two groups.

Lastly, we assessed for differences in type of reconstruction (primary vs. secondary) and for differences in outcomes based on the occurrence of complications requiring surgery. No differences in satisfaction were found between patients with primary versus secondary BR (data not shown). In addition, no statistically significant differences were found in patients who suffered complications (any type) requiring additional surgery (data not shown).

\section{Discussion}

PROMs are becoming increasingly relevant for the assessment of surgical quality in BR and to help guide clinical decision-making $[12,15]$. The aim of this study was to evaluate QoL and satisfaction with outcomes over longer periods of time in postmastectomy patients who underwent free-flap BR. Overall, there is moderate evidence to support that patients who underwent BR longer ago have higher overall satisfaction with outcomes and with their breasts compared to patients who underwent surgery more recently [21]. In patients who were treated more than 5 years prior, psychosocial well-being was also found to be better when compared to patients treated more recently.

Although this study is limited by the low number of responders and by the fact that we did not adjust for multiple testing due to the explorative nature of the analyses, 


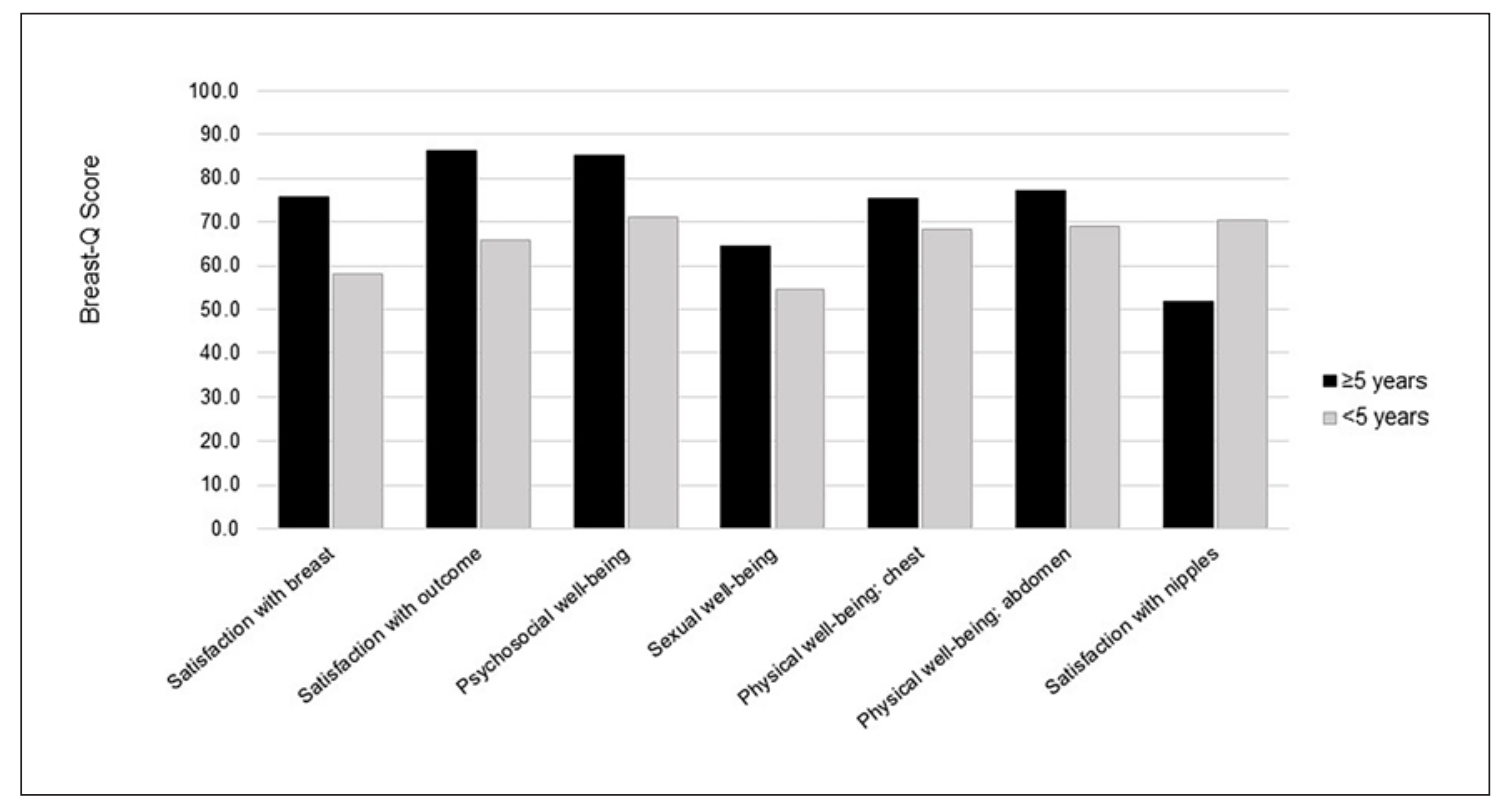

Fig. 2. BREAST-Q scores by time since free-flap breast reconstruction.

Table 3. BREAST-Q scores by time since free-flap breast reconstruction

\begin{tabular}{llll}
\hline BREAST-Q domains & \multicolumn{2}{l}{ Time since breast reconstruction } & \multirow{2}{*}{$p$ value } \\
\cline { 2 - 3 } & $\geq 5$ years & $<5$ years & \\
\hline Satisfaction with breast & $76.60(13.77)$ & $59.70(16.61)$ & 0.006 \\
Satisfaction with outcome & $88.60(12.46)$ & $66.94(21.78)$ & 0.005 \\
Psychosocial well-being & $87.80(15.53)$ & $72.03(17.94)$ & 0.016 \\
Sexual well-being & $68.10(18.23)$ & $54.87(23.25)$ & 0.11 \\
Physical well-being: chest & $77.10(20.59)$ & $68.42(17.57)$ & 0.20 \\
Physical well-being: abdomen & $76.56(26.27)$ & $69.77(23.25)$ & 0.46 \\
Satisfaction with nipples & $54.00(33.26)$ & $66.88(29.14)$ & 0.36 \\
\hline
\end{tabular}

Data are presented as mean (standard deviation). we observed improved satisfaction with outcomes and with breasts as time since BR progressed in both linear regression and grouped analyses. The higher satisfaction with breast scores reported by patients treated longer ago suggests that satisfaction and QoL have the potential to improve as time passes. Prior studies have also shown that QoL improves over time in cancer patients $[22,23]$ and also specifically after BR [24]. Our study parallels the findings by Nelson and colleagues [25] who also studied long-term satisfaction and QoL outcomes in patients who underwent autologous and implant-based $\mathrm{BR}$ at a single academic institution in the USA [25]. We found no differences in satisfaction outcomes between patients who underwent primary versus secondary BR. Although the majority of patients undergo primary BR due to better aesthetic results, secondary BR is sometimes preferred or required in selected patients. A previous study by Yoon and colleagues [26] investigated PROMs in immediate and delayed BR and found no effect of timing of BR on QoL and satisfaction outcomes, even though preoperative PROMs differed significantly. These findings suggest that it is the BR itself which instigates an enhanced QoL and continues to improve over time. Long-term QoL in cancer survivors has even been reported to reach levels comparable to general population in multiple studies [23, $27,28]$. An explanation for Improved outcomes may also be associated with a response shift over time: as time passes, disease-free survivors have a more pragmatic view of the role of cancer in their everyday life [22]. The improved satisfaction with outcomes and breasts that we observed suggests that the surgery itself may not be the only relevant factor determining postoperative QoL and satisfaction. Psychological factors may also influence QoL and satisfaction, including the time that women need to adapt to the new body, as some women may feel disconnected to their breast(s) $[4,29]$. 
Another important factor in patient well-being in the context of disease is coping. Healthy coping mechanisms such as self-regulation abilities and prevention of avoidance can improve QoL over time [29]. With time progressing after surgery, women may experience cognitive and emotional processing, which can lead to posttraumatic growth and therefore improved QoL in patients after breast reconstructive surgery [30]. The current study showed better psychosocial well-being, but not sexual well-being, in patients who underwent BR longer ago, which again is similar to the results demonstrated in the study by Nelson and colleagues [25]. Psychological interventions can have a positive impact on posttraumatic growth, thereby leading to better QoL [29]. It is well known that a breast cancer diagnosis can be a traumatic event and needs to be approached in a multidisciplinary manner [29]. In recent years, focus on psychological care for breast cancer patients has become increasingly evident and the effect of specific interventions has led to better psychological well-being in breast cancer patients [31].

In conclusion, time since BR resulted in improved PROMs such as QoL and satisfaction in patients. The current study underlines the importance of long-term PROMs related to breast cancer treatment and BR, to help provide patients and health care professionals with a realistic outlook and to facilitate managing expectations regarding $\mathrm{BR}$.

\section{Acknowledgement}

All listed authors contributed to the research and/or to the manuscript. We would like to thank all women who participated in this study for their valuable contributions.

\section{Statement of Ethics}

The study protocol was approved by the Swiss Ethics Committees on research involving humans (BASEC No. 2018-00867). The study was designed in accordance with the EQUATOR Network's recommendations on reporting patient-reported outcomes in clinical trials. All patients have given their written informed consent.

\section{Conflict of Interest Statement}

The authors have no conflicts of interest to declare.

\section{Funding Sources}

A.L.P. is a co-developer of the BREAST-Q and receives royalties when it is used in for-profit, industry-sponsored clinical trials.

\section{Author Contributions}

D.B.Y.F. contributed to the design, coordination, execution, data analysis, and publication of this study. M.O. contributed to the execution of this study and writing of the manuscript. D.B.Y.F. and M.O. contributed equally. L.H. contributed to the data analysis and editing of the manuscript. P.G. contributed to the editing and publication of the manuscript. A.L.P. contributed to the design of the questionnaires and editing of the manuscript. N.L. contributed to the treatment of patients, coordination of the study, and publication of the manuscript. All authors read and approved the final version of the manuscript.

\section{Data Availability Statement}

All data that support the findings of this study can be made available on request to the corresponding author.

\section{References}

1 WHO. Breast Cancer: Prevention and Control 2020 [available from: https://www.who. int/cancer/detection/breastcancer/en/index1.html.

2 Matthews H, Carroll N, Renshaw D, Turner A, Park A, Skillman J, et al. Predictors of satisfaction and quality of life following postmastectomy breast reconstruction. Psychooncology. 2017;26(11):1860-5.

3 Beugels J, Kool M, Hoekstra LT, Heuts EM, Tuinder SMH, van der Hulst RRWJ, et al. Quality of Life of Patients After Immediate or Delayed Autologous Breast Reconstruction: A Multicenter Study. Ann Plast Surg. 2018; 81(5):523-7.

4 Matthews H, Turner A, Williamson I, Clyne W. 'It's a silver lining': A template analysis of satisfaction and quality of life following postmastectomy breast reconstruction. $\mathrm{Br} \mathrm{J}$ Health Psychol. 2018;23(2):455-75.
5 Al-Ghazal SK, Fallowfield L, Blamey RW. Comparison of psychological aspects and patient satisfaction following breast conserving surgery, simple mastectomy and breast reconstruction. Eur J Cancer. 2000;36(15): 1938-43.

6 Thorarinsson A, Fröjd V, Kölby L, Ljungdal J, Taft C, Mark H. Long-Term Health-Related Quality of Life after Breast Reconstruction: Comparing 4 Different Methods of Reconstruction. Plast Reconstr Surg Glob Open. 2017;5(6):e1316.

7 Cordova LZ, Hunter-Smith DJ, Rozen WM. Patient reported outcome measures (PROMs) following mastectomy with breast reconstruction or without reconstruction: a systematic review. Gland Surg. 2019;8(4):441-51.

8 Cano SJ, Browne JP, Lamping DL. Patientbased measures of outcome in plastic surgery: current approaches and future directions. $\mathrm{Br}$ J Plast Surg. 2004;57(1):1-11.
9 Colakoglu S, Khansa I, Curtis MS, Yueh JH, Ogunleye A, Haewyon C, et al. Impact of complications on patient satisfaction in breast reconstruction. Plast Reconstr Surg. 2011; 127(4):1428-36.

10 Berry DA, Cronin KA, Plevritis SK, Fryback DG, Clarke L, Zelen M, et al. Effect of screening and adjuvant therapy on mortality from breast cancer. N Engl J Med. 2005;353(17): 1784-92.

11 Wood SH, Tarar MN. Outcome audit in plastic surgery: the Cambridge Classification. $\mathrm{Br} \mathrm{J}$ Plast Surg. 1994;47(2):122-6.

12 Cano SJ, Klassen AF, Scott AM, Pusic AL. A closer look at the BREAST-Q(@). Clin Plast Surg. 2013;40(2):287-96.

13 Cano SJ, Klassen A, Pusic AL. The science behind quality-of-life measurement: a primer for plastic surgeons. Plast Reconstr Surg. 2009; 123(3):98e-106e. 
14 Elder EE, Brandberg Y, Björklund T, Rylander R, Lagergren J, Jurell G, et al. Quality of life and patient satisfaction in breast cancer patients after immediate breast reconstruction: a prospective study. Breast. 2005;14(3):201-8.

15 Pusic AL, McCarthy C, Cano SJ, Klassen AF, Kerrigan CL. Clinical research in breast surgery: reduction and postmastectomy reconstruction. Clin Plast Surg. 2008;35(2):215-26.

16 Wilkins EG, Cederna PS, Lowery JC, Davis JA, Kim HM, Roth RS, et al. Prospective analysis of psychosocial outcomes in breast reconstruction: one-year postoperative results from the Michigan Breast Reconstruction Outcome Study. Plast Reconstr Surg. 2000; 106(5):1014-25, discussion 1026-7.

17 Gopie JP, Timman R, Hilhorst MT, Hofer SO Mureau MA, Tibben A. The short-term psychological impact of complications after breast reconstruction. Psychooncology. 2013; 22(2):290-8.

18 Lu SM, Nelson JA, Fischer JP, Fosnot J, Goldstein J, Selber JC, et al. The impact of complications on function, health, and satisfaction following abdominally based autologous breast reconstruction: a prospective evaluation. J Plast Reconstr Aesthet Surg. 2014; 67(5):682-92
19 Alderman AK, Kuhn LE, Lowery JC, Wilkins EG. Does patient satisfaction with breast reconstruction change over time? Two-year results of the Michigan Breast Reconstruction Outcomes Study. J Am Coll Surg. 2007; 204(1):7-12.

20 Calvert M, Kyte D, Mercieca-Bebber R, Slade A, Chan AW, King MT, et al. Guidelines for Inclusion of Patient-Reported Outcomes in Clinical Trial Protocols: The SPIRIT-PRO Extension. JAMA. 2018;319(5):483-94.

21 Bland M. An Introduction to Medical Statistics. 4th ed. Oxford University Press; 2015.

22 Klein D, Mercier M, Abeilard E, Puyraveau M, Danzon A, Dalstein V, et al. Long-term quality of life after breast cancer: a French registry-based controlled study. Breast Cancer Res Treat. 2011;129(1):125-34.

23 Hsu T, Ennis M, Hood N, Graham M, Goodwin PJ. Quality of life in long-term breast cancer survivors. J Clin Oncol. 2013;31(28): 3540-8.

24 Hunsinger V, Hivelin M, Derder M, Klein D, Velten M, Lantieri L. Long-Term Follow-Up of Quality of Life following DIEP Flap Breast Reconstruction. Plast Reconstr Surg. 2016; 137(5):1361-71.

25 Nelson JA, Allen RJ, Polanco T, Shamsunder M, Patel AR, McCarthy CM, et al. Long-term Patient-reported Outcomes Following Postmastectomy Breast Reconstruction: An 8-year Examination of 3268 Patients. Ann Surg. 2019;270(3):473-83.
26 Yoon AP, Qi J, Brown DL, Kim HM, Hamill JB, Erdmann-Sager J, et al. Outcomes of immediate versus delayed breast reconstruction: Results of a multicenter prospective study. Breast. 2018;37:72-9.

27 Doege D, Thong MS, Koch-Gallenkamp L, Bertram H, Eberle A, Holleczek B, et al. Health-related quality of life in long-term disease-free breast cancer survivors versus female population controls in Germany. Breast Cancer Res Treat. 2019;175(2):499-510.

28 Helgeson VS, Tomich PL. Surviving cancer: a comparison of 5-year disease-free breast cancer survivors with healthy women. Psychooncology. 2005;14(4):307-17.

29 Pat-Horenczyk R, Perry S, Hamama-Raz Y, Ziv Y, Schramm-Yavin S, Stemmer SM. Posttraumatic Growth in Breast Cancer Survivors: Constructive and Illusory Aspects. J Trauma Stress. 2015;28(3):214-22.

30 İnan FŞ, Üstün B. Breast Cancer and Posttraumatic Growth. J Breast Health. 2014; 10(2):75-8.

31 Guarino A, Polini C, Forte G, Favieri F, Boncompagni I, Casagrande M. The Effectiveness of Psychological Treatments in Women with Breast Cancer: A Systematic Review and Meta-Analysis. J Clin Med. 2020;9(1):209. 